\title{
Two-loop Splitting Amplitudes
}

\author{
Z. Bern ${ }^{a *}$, L.J. Dixon ${ }^{\mathrm{b} \dagger}$ and D.A. Kosower ${ }^{\mathrm{c}}$ \\ ${ }^{\text {a}}$ Department of Physics and Astronomy, UCLA \\ Los Angeles, CA 90095-1547, USA \\ ${ }^{\mathrm{b}}$ Stanford Linear Accelerator Center, Stanford University \\ Stanford, CA 94309, USA \\ c Service de Physique Théorique, CEA-Saclay \\ F-91191 Gif-sur-Yvette cedex, France
}

Splitting amplitudes govern the behavior of scattering amplitudes at the momenta of external legs become collinear. In this talk we outline the calculation of two-loop splitting amplitudes via the unitarity sewing method. This method retains the simple factorization properties of light-cone gauge, but avoids the need for prescriptions such as the principal value or Mandelstam-Leibbrandt ones. The encountered loop momentum integrals are then evaluated using integration-by-parts and Lorentz invariance identities. We outline a variety of applications for these splitting amplitudes.

\section{INTRODUCTION}

Recent years have seen rapid progress in computing higher-order corrections to the standard model. In particular, there have been a large number of new computations of two-loop scattering amplitudes with more than a single kinematic variable (see refs. [1] and references therein.). In the past decades, a number of new approaches have been developed to cope with this complexity, including helicity methods, color decompositions, recursion relations, ideas based on string theory, and the unitarity-based method, summarized in a variety of review articles [2]. Much of the progress at two loops has been facilitated by new techniques for performing loop integrals (see refs. [3] ).

Splitting amplitudes describe the universal singular behavior of amplitudes in the regions of phase space where momenta become collinear. In this talk we summarize the calculation of $g \rightarrow g g$

\footnotetext{
*Presenter at 7th DESY Workshop on Elementary Particle Theory, Loops and Legs in Quantum Field Theory, April 25-30, Zinnowitz (Usedom Island), Germany. Research supported by the US Department of Energy under contract DE-FG03-91ER40662.

${ }^{\dagger}$ Research supported by the US Department of Energy un-
} der contract DE-AC03-76SF00515. splitting amplitudes at two loops via the unitarity sewing method [4]. The two-loop splitting amplitudes, including the ones involving quarks, have also been extracted recently from explicit computations of four-point helicity amplitudes with a massive leg [ [5]. With the unitarity sewing method the simple factorization properties of light-cone gauge are retained, but the need for principal value or other such prescriptions [ [ 6 ] is avoided.

We also discuss a variety of applications of twoloop splitting amplitudes. These include verifying amplitude calculations [ 2] or constructing ansätze [ 7, 8] for amplitudes with more than two kinematic variables. Splitting amplitudes also enter into an alternative method [9] for computing corrections to the DGLAP kernel governing the $Q^{2}$ evolution of parton distributions and fragmentation functions. We have also used them in a proof [4] of Catani's formula [10] for universal two-loop infrared divergences, complementing the proof based on resummation [11]. They have also been used to provide direct evidence that in maximally supersymmetric $\mathcal{N}=4$ super-Yang-Mills theory, higher-loop planar amplitudes can be expressed in terms of lower-loop ones, suggesting that substantial portions of the theory may be 


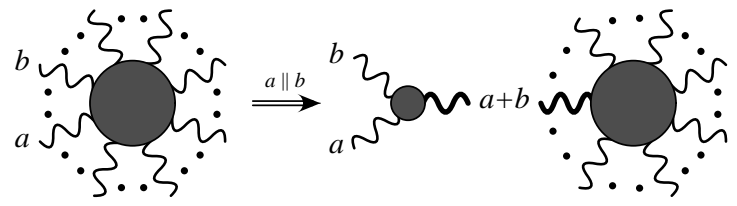

Figure 1. The collinear factorization of a treelevel amplitude. The thick line represents a slightly off-shell gluon.

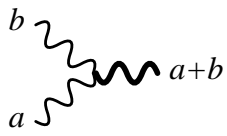

Figure 2. The three-point vertex diagram for obtaining the tree-level splitting amplitude.

solvable.

\section{SPLITTING AMPLITUDES}

For simplicity, here we consider only the leading color contributions. The full color structure of the two-loop splitting amplitudes may be found in refs. [4, 5]. At $L$ loops the behavior of the leading-color contributions are given by [ 2, 7, 8, 12, 13,

$A_{n}^{(L)}\left(\ldots, a^{\lambda_{a}}, b^{\lambda_{b}}, \ldots\right) \stackrel{a \| b}{\longrightarrow}$

$\sum_{l=0}^{L} \sum_{\lambda= \pm} \operatorname{Split}_{-\lambda}^{(l)}\left(z ; a^{\lambda_{a}}, b^{\lambda_{b}}\right) A_{n-1}^{(L-l)}\left(\ldots, P^{\lambda}, \ldots\right)$,

in the limit where the momenta $k_{a} \rightarrow z k_{P}$ and $k_{b} \rightarrow(1-z) k_{P}$ with $k_{P}=k_{a}+k_{b}$. Here Split $_{-\lambda}^{(l)}\left(z ; a^{\lambda_{a}}, b^{\lambda_{b}}\right)$ is an $l$-loop splitting amplitude. Legs $a$ and $b$ carry helicities $\lambda_{a}$ and $\lambda_{b}$, while the merged leg $P$ carries helicity $\lambda$, runs over the two helicities of the intermediate state. In the other sum, $l$ runs over the loop order. As a simple example, the collinear factorization of an $n$-point tree amplitude into a splitting amplitude and an $(n-1)$-point amplitude is depicted schematically in fig. [1]

At tree level, the splitting amplitudes are rather straightforward to obtain by evaluating diagrams. The splitting amplitude depicted in fig. [1 may be computed directly from the Feynman three-vertex depicted in fig. 2 2 by multiplying by the off-shell propagator and saturating the external legs with helicity states [2].

Beyond tree level the analogous computation is more subtle. In covariant gauges there are additional 'non-factorizing' contributions associated with soft-gluon emission, complicating the analysis [ 14]. One might be tempted to avoid this difficulty by using instead physical gauges such as light-cone gauge since they have simple factorization properties. Unfortunately, light-cone gauge has another set of complications arising from the propagator,

$$
D_{\mu \nu}=-\frac{i}{p^{2}+i \epsilon}\left[\eta_{\mu \nu}-\frac{n_{\mu} p_{\nu}+p_{\mu} n_{\nu}}{p \cdot n}\right],
$$

where $p$ is the particle momentum, $\eta_{\mu \nu}$ is the Minkowski metric, and $n$ is a null vector $\left(n^{2}=0\right)$ defining the light-cone direction. The problem occurs in the region where $p \cdot n$ vanishes. One common method for dealing with this singularity is the principal-value prescription which replaces

$$
\frac{1}{p \cdot n} \rightarrow \lim _{\delta \rightarrow 0} \frac{1}{2}\left(\frac{1}{p \cdot n+i \delta}+\frac{1}{p \cdot n-i \delta}\right),
$$

where $\delta$ is a regulator parameter. Another choice, better founded in field theory, is the MandelstamLeibbrandt prescription [ [6]. The introduction of any of these prescriptions in a splitting amplitude calculation is problematic for a number of reasons. The most serious problem is that results retain dependence on the regulator parameter $\delta$. (In general these cancel only after combining virtual and real emission contributions.) A calculation of the splitting amplitudes that retains this dependence cannot match the splitting amplitudes extracted from the collinear limits of scattering amplitudes which are independent of such a parameter. Thus one would not obtain the desired results.

Is the need for these prescriptions is an artifact of the gauge choice? Could they be avoided in a more physical construction of the splitting amplitudes? Yes! As a concrete example, consider the two integrals in fig. 3 In a light-cone gauge calculation of the one-loop $g \rightarrow g g$ splitting amplitudes both integrals appear. What distinguishes 


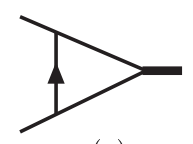

(a)

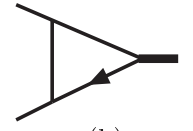

(b)
Figure 3. One-loop triangle diagrams containing light-cone denominators indicated by the arrow. Only integral (b) appears in the unitarity sewing method.

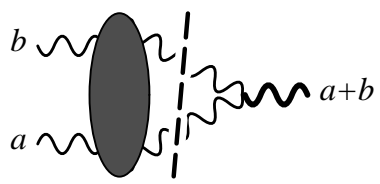

Figure 4. The two-particle cut of a one-loop splitting amplitude. The cut is represented by the dashed line. On the left-hand side all legs, including the cut ones, are on-shell.

these two integrals? The key is the unitarity cut shown in fig. 4 On the left-hand-side of the cut is an on-shell gauge invariant amplitude which does not contain light-cone denominators. This leads one to suspect that the integral in fig. 4(a) is spurious and should not appear. On the other hand, the light-cone denominator in integral (b) does appear in the physical state projectors of the unitarity cut suggesting that it cannot be removed. Integral (b), however, is perfectly well defined using dimensional regularization and is therefore harmless.

\section{CONSTRUCTION AND RESULTS}

To avoid the appearance of ill-defined integrals we use the unitarity sewing method [ 8, 15, 13, 4. With this method the loop integrands are obtained algorithmically by constructing unrestricted loop momentum integrals with the correct cuts in all channels. The method is welltested, having been applied to a variety of problems. For example it has been used to obtain analytic formulas for $Z \rightarrow 4$ partons at one loop [16] and one-loop maximally helicity violating (MHV) amplitudes with an arbitrary number of external legs in supersymmetric gauge theories [8]. More relevant for the discussion here, it has also been used to obtain one-loop splitting amplitudes [13 .

The two-loop splitting amplitudes for $g \rightarrow g g$ were constructed by combining the three contributing unitarity cuts shown in fig. 5 into a single integrand [4]. One simplifying feature of the $g \rightarrow g g$ splitting amplitudes is that the nonplanar configurations do not contribute, because of color considerations. The resulting integrand was then evaluated in terms of 13 master integrals using the implementation of the Laporta algorithm [17] due to Anastasiou and Lazopoulos [ 18. The master integrals were computed by constructing and solving a set of differential equations, along the lines of refs. [19].

The results for the two-loop QCD splitting amplitudes are too lengthy to include here, but are given in detail in ref. [ 4 where they are expressed in terms in terms of a series expansion in $\epsilon$, with each term containing ordinary logarithms and polylogarithms.

The two-loop results for $\mathcal{N}=4$ super-YangMills theory have an especially intriguing feature. To expose this, it is convenient to first write the loop splitting amplitudes in terms of a ratio with the corresponding tree functions,

$$
\begin{aligned}
& \operatorname{Split}_{-\lambda}^{(L)}\left(a^{\lambda_{a}}, b^{\lambda_{b}}\right) \\
& \quad=r_{S}^{(L) \lambda_{a}, \lambda_{b}}\left(z, s_{a b}\right) \times \operatorname{Split}_{-\lambda}^{(0)}\left(a^{\lambda_{a}}, b^{\lambda_{b}}\right),
\end{aligned}
$$

where $L$ indicates the loop order. Rather surprisingly the two-loop ratios are expressible in terms of the one-loop ratios [ 20$]$,

$$
\begin{aligned}
r_{S}^{(2), \mathcal{N}=4}(\epsilon ; z, s) & =\frac{1}{2}\left(r_{S}^{(1), \mathcal{N}=4}(\epsilon ; z, s)\right)^{2} \\
& +f(\epsilon) r_{S}^{(1), \mathcal{N}=4}(2 \epsilon ; z, s)+\mathcal{O}(\epsilon) .
\end{aligned}
$$

The explicit values of $r_{S}^{(1), \mathcal{N}=4}, r_{S}^{(2), \mathcal{N}=4}$ and $f(\epsilon)$ may be found in refs. [ 20,4$]$. This relation between the two-loop and one-loop splitting amplitudes may provide a hint to unraveling the theory.

\section{APPLICATIONS}

One use of splitting amplitudes is to provide checks on calculations of amplitudes with more than two kinematic invariants from the collinear constraints [ 2]. At two loops the $g \rightarrow g g$ amplitudes are consistent with the collinear limits 

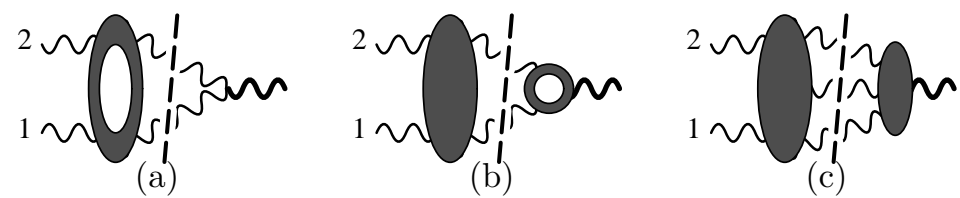

Figure 5. The three cuts contributing to the two-loop splitting amplitude.

of the two-loop $H \rightarrow g g g$ helicity amplitudes [ 21, 4, 5. In some cases the collinear constraints are sufficient for constructing ansätze for $n$-point scattering amplitudes [ [7, 8]. It is worth noting that after subtracting the divergences from an amplitude, the finite remainders also satisfy universal collinear constraints [4], providing a direct check on these terms.

Another application of the two-loop splitting amplitudes is for a proof of Catani's formula [ 10] for two-loop infrared divergences [4]. The proof is based on taking collinear limit of the $n$ point formula, and comparing it with the $(n-1)$ point formula. The proof does require the reasonable assumption that terms do not vanish in all collinear limits. This proof is complementary to the one based on resummation [11], since it fixes the explicit form of the functions appearing in Catani's formula, including the previously unknown $n$-point color non-trivial terms at order $1 / \epsilon$.

The two-loop splitting amplitudes are also one of the ingredients to an alternative approach [ 9 to computing the DGLAP kernels at NNLO. These kernels have recently been computed by Moch, Vermaseren and Vogt [22] using the Mellin space approach of computing anomalous dimensions of leading-twist operators. The computation of these kernels are of great importance for precision extraction of parton distribution functions from experimental data. As discussed in ref. [4, the splitting amplitudes can straightforwardly be continued to the space-like region relevant for the evolution of parton distribution functions.

A more theoretical application of splitting amplitudes is for investigating the form of the multiloop scattering amplitudes in maximally supersymmetric Yang-Mills theory. It has been 30 years since 't Hooft suggested that QCD could be solved in the planar limit. Unfortunately, this has not been achieved as yet, so a logical approach is to start with a simpler theory. The structure of maximally supersymmetric $\mathcal{N}=4$ YangMills theory is much simpler than QCD since it possesses superconformal symmetry. Moreover, the Maldacena conjecture [ 23] suggests that the strongly coupled limit of the theory should be much simpler than one might otherwise expect, since it is dual to weakly coupled gravity in antide Sitter space.

The two-loop splitting amplitudes and their associated collinear constraints lead to an ansatz for the planar contributions to the $n$-point two-loop amplitudes in terms of the $n$-point one-loop amplitudes of the form [20]

$$
\begin{gathered}
M_{n}^{(2)}(\epsilon)=\frac{1}{2}\left(M_{n}^{(1)}(\epsilon)\right)^{2}+f(\epsilon) M_{n}^{(1)}(2 \epsilon) \\
-\frac{5}{4} \zeta_{4}+\mathcal{O}(\epsilon),
\end{gathered}
$$

where $f(\epsilon)$ is the same function as appearing in eq. (11). The $M_{n}^{(L)}(\epsilon)$ are $n$-point $L$-loop planar amplitudes, divided by the tree amplitudes. For $n=4$ explicit calculation [ [24, 25, 20] confirms this ansatz. More generally, for $n \geq 5$ the ansatz (2) satisfies the correct collinear properties, using the splitting amplitudes (1). For the maximally helicity-violating amplitudes it is likely that the ansatz (2) is correct, but for the nonmaximally helicity violating amplitudes it would be important to also check multi-particle factorization. The crucial question is whether this iterative structure holds to higher loops and whether a resummation is possible. An important step to investigate the structure would be the computation of the three-loop four-point amplitude. This computation seems feasible [26], especially since one of two required integrals [ 24] has already been evaluated by Smirnov [27]. 


\section{REFERENCES}

1. T. Gehrmann, hep-ph/0211169 Int. J. Mod. Phys. A19, 851 (2004) hep-ph/0310178; E.W.N. Glover, Nucl. Phys. Proc. Suppl. 116, 3 (2003) hep-ph/0211412; Z. Bern, Nucl. Phys. Proc. Suppl. 117, 260 (2003) hep-ph/0212406.

2. M.L. Mangano and S.J. Parke, Phys. Rept. 200, 301 (1991); L.J. Dixon, hep-ph/9601359

Z. Bern, L.J. Dixon and D.A. Kosower, Ann. Rev. Nucl. Part. Sci. 46, 109 (1996) hep-ph/9602280.

3. T. Gehrmann and E. Remiddi, hep-ph/0101147. P. Mastrolia and E. Remiddi, Nucl. Phys. Proc. Suppl. 116, 412 (2003) hep-ph/0211210; V.A. Smirnov, Nucl. Phys. Proc. Suppl. 116, 417 (2003) hep-ph/0209295.

4. Z. Bern, L.J. Dixon and D.A. Kosower, hep-ph/0404293.

5. S.D. Badger and E.W.N. Glover, hep-ph/0405236

6. S. Mandelstam, Nucl. Phys. B213, 149 (1983); G. Leibbrandt, Noncovariant Gauges: Quantization of Yang-Mills and Chern-Simons Theory in Axial-Type Gauges, World Scientific (1994), Singapore.

7. S.J. Parke and T.R. Taylor, Phys. Rev. Lett. 56, 2459 (1986); Z. Bern, G. Chalmers, L.J. Dixon and D.A. Kosower, Phys. Rev. Lett. 72, 2134 (1994) hep-ph/9312333.

8. Z. Bern, L.J. Dixon, D.C. Dunbar and D.A. Kosower, Nucl. Phys. B425, 217 (1994) hep-ph/9403226; Z. Bern, L.J. Dixon, D.C. Dunbar and D.A. Kosower, Nucl. Phys. B435, 59 (1995) hep-ph/9409265.

9. D.A. Kosower and P. Uwer, in proceedings of International Europhysics Conference on High-Energy Physics (EPS-HEP 99), Tampere, Finland (1999); Nucl. Phys. B674, 365 (2003) hep-ph/0307031; D. de Florian and M. Grazzini, Nucl. Phys. B616, 247 (2001) hep-ph/0108273.

10. S. Catani, Phys. Lett. B427, 161 (1998) hep-ph/9802439.

11. G. Sterman and M.E. Tejeda-Yeomans, Phys. Lett. B552, 48 (2003) hep-ph/0210130.
12. Z. Bern, V. Del Duca and C.R. Schmidt, Phys. Lett. B445, $168 \quad$ (1998) hep-ph/9810409; Z. Bern, V. Del Duca, W.B. Kilgore and C.R. Schmidt, Phys. Rev. D 60, 116001 (1999) hep-ph/9903516; S. Catani and M. Grazzini, Phys. Lett. B446, 143 (1999) hep-ph/9810389; Nucl. Phys. B570, 287 (2000) hep-ph/9908523.

13. D. A. Kosower, Nucl. Phys. B552, 319 (1999) hep-ph/9901201; D.A. Kosower and P. Uwer, Nucl. Phys. B 563, 477 (1999) hep-ph/9903515.

14. Z. Bern and G. Chalmers, Nucl. Phys. B 447, 465 (1995) hep-ph/9503236.

15. Z. Bern and A.G. Morgan, Nucl. Phys. B 467, 479 (1996) hep-ph/9511336;

Z. Bern, L.J. Dixon and D.A. Kosower, Ann. Rev. Nucl. Part. Sci. 46, 109 (1996) hep-ph/9602280.

16. Z. Bern, L.J. Dixon and D.A. Kosower, Nucl. Phys. B 513, 3 (1998) hep-ph/9708239.

17. S. Laporta, Int. J. Mod. Phys. A15, 5087 (2000) hep-ph/0102033.

18. C. Anastasiou and A. Lazopoulos, hep-ph/0404258

19. V.A. Smirnov and O.L. Veretin, Nucl. Phys. B566, 469 (2000) hep-ph/9907385; T. Gehrmann and E. Remiddi, Nucl. Phys. B 580, 485 (2000) hep-ph/9912329.

20. C. Anastasiou, Z. Bern, L.J. Dixon and D.A. Kosower, Phys. Rev. Lett. 91, 251602 (2003) hep-th/0309040.

21. A. Koukoutsakis, Ph.D. thesis, U. Durham (2003).

22. S. Moch, J.A.M. Vermaseren and A. Vogt, hep-ph/0403192, hep-ph/0404111.

23. J.M. Maldacena, Adv. Theor. Math. Phys. 2, 231 (1998) hep-th/9711200; S.S. Gubser, I.R. Klebanov and A.M. Polyakov, Phys. Lett. B 428, 105 (1998) hep-th/9802109.

24. Z. Bern, J.S. Rozowsky and B. Yan, Phys. Lett. B 401, 273 (1997) hep-ph/9702424.

25. V.A. Smirnov, Phys. Lett. B 460, 397 (1999) hep-ph/9905323.

26. V. A. Smirnov, hep-ph/0406052.

27. V.A. Smirnov, Phys. Lett. B 567, 193 (2003) hep-ph/0305142. 